\title{
EFFECT OF UNSATURATED POLYESTER CONCENTRATION ON RHEOLOGICAL CHARACTERISTICS OF POLYPROPYLENE-UNSATURATED POLYESTER REACTIVE BLEND
}

\author{
G. M. Mamoor, M. N. Khan*, A. Ali, S. Shahid, M. Z. Iqbal \\ Department of Polymer \& Process Engineering, University of Engineering \& Technology Lahore, Pakistan
}

Received 28 August 2010; received in revised form 5 December 2010

\begin{abstract}
Reactive blends of polypropylene and unsaturated polyester with different compositions were prepared by melt processing using dicumyl peroxide (DCP) as initiator in a plasticoder. Rheological Characteristics of the blends were studied as a function of unsaturated polyester concentration over a range of strain rate. The possibility of forming a graft polymer increased with increasing the unsaturated polyester content. Change in viscosity behavior of the blends was a result of the competing reactions of degradation and grafting.
\end{abstract}

Keywords: DCP, strain rate, degradation, grafting

DOI:10.3329/cerb.v14i2.5955

\section{Introduction}

Physical blending has been exercised as an effective mean to alter the properties of already available polymers [1]. Interfacial interaction and strength are the deciding factors in the making of products from these blends. Reactive compatibilization has proved to be more effective than physical and improves the interfacial properties along with stabilized and controlled morphology [2].

PET is one of the most widely used engineering thermoplastics but its moderate impact strength limits its use in many of engineering applications. Many attempts has been made to improve its impact strength by blending it with olefins [3], Ethylene Propylene Rubber [4], Ethylene Propylene Diene Monomer [5], Maleic anhydride grafted rubber [6] and many more. However, PET with reactive compatibilized polypropylene-unsaturated polyester (PP-UP) blend as a compatibilizer for rubber phase has established its importance.

It is well recognized that the rheological behavior of the blend is dependent on molecular structure [7]. The stressstrain rate relationship of polymeric fluids is best described by mathematical models known as constitutive equations. Different constitutive equations are developed because of the complex chain structure and viscoelastic behavior of polymers but only few are satisfactory. For general flow of polymer fluids, following equation is generally valid

$$
\tau=\eta(T, \gamma, P) \gamma^{n}
$$

where, $\tau$ is the shear stress, $\gamma$ is the strain rate and $\eta$ is viscosity that is function of pressure, temperature and strain rate. It can be noted from the above equation that constitutive equation for various fluids at different conditions can be derived if corresponding viscosity expressions are substituted

*Corresponding author, Email:naveedkhan1975@yahoo.com into it. Development of viscosity models that represent the viscosity and relevant parameters, including molecular structure parameters and processing parameters to predict the flow properties, is one of the targets for rheologists and engineers. Hence, a number of viscosity models were developed [8-10].

In present work, PP-UP (Polypropylene-Unsaturated polyester) blends were prepared by melt blending of unsaturated polyester with polypropylene in different compositions in the presence of peroxide initiator and Cobalt Octoate salt solution as a promoter. Blending was carried out in a plasticoder at elevated temperature. Chemical modification of PP-UP blend resulted in improved properties than the nonreactive blend [11]. In this study, the change in pseudoplastic behavior of the blend is investigated as a function of UP (unsaturated polyester) concentration and strain rate. Power law parameters are calculated for different blend compositions. Bagley correction is also applied to the system.

\section{Theory}

In this work, the flow of polymer through a circular die is studied. For general case of fully developed region with no wall slip, the shear stress at the wall is calculated by

$$
\tau=\frac{\left(P_{1}-P_{0}\right) R}{2 L}
$$

where $P_{l}$ is pressure drop through the die and $P_{0}$ is the pressure drop through an orifice (with $L / R<1$ ) under the same conditions. The apparent shear viscosity is given by the following equation

$$
\eta=\frac{\tau}{\gamma}
$$

and the apparent shear rate is given by

$$
\gamma=\frac{4 Q}{\pi R^{3}}
$$

(C)2010 Bangladesh Uni. of Engg. \& Tech. 
Table 1: Blend compositions

\begin{tabular}{lcccc}
\hline Blend compostion $(\%)$ & $80 / 20$ & $60 / 40$ & $40 / 60$ & $30 / 70$ \\
\hline PP $(\mathrm{g})$ & 40 & 30 & 20 & 15 \\
UP $(\mathrm{g})$ & 10 & 20 & 30 & 35 \\
DCP $(\mathrm{g})$ & 0.2 & 0.2 & 0.2 & 0.2 \\
Cobalt salt solution $(\mathrm{ml})$ & 1.5 & 1.5 & 1.5 & 1.5 \\
\hline
\end{tabular}

where $Q$ is the volumetric flow rate.

The values of shear stress and apparent shear rate are measured during flow of polymer through die. Thus, the melt viscosity can be determined from Equation 3. Melt viscosity calculated in this way can be plotted against shear rate to get melt viscosity- shear rate curve. It can be seen from the curve that change of viscosity with strain rate is not significant at low strain rate for non Newtonian fluids. But it shows a drastic change over a moderate range of strain rate and then again a constant region at higher strain rate is observed [12].

\section{Experimental}

\subsection{Materials}

Basic materials used in present work are polypropylene and unsaturated polyester. PP (Polypropylene) was purchased from Reliance Company limited Jamnagar Gujrat. UP (unsaturated polyester) was purchased from Descon Chemicals Limited, Pakistan. Both are commercial grade. DCP was imported from Sigma Aldrich Cheme Stelnheim Germany.

\subsection{Blend preparation}

PP-UP blends are generally prepared by reactive blending techniques. For the present work, blends were prepared by melt mixing of polypropylene with unsaturated polyester using DCP as an initiator and cobalt octoate solution as promoter. Blending was carried out in a Brabender plasticoder (PLE-330) at a temperature of $180^{\circ} \mathrm{C}$ and at a rotor speed of 80 revolutions per minute. Mixing was continued for 5 minutes. During blend formation, the following sequence was adopted to prepare the blends of different composition. PP was introduced first into the mixer followed by UP. After the complete melting of the components, DCP was added to initiate the reaction. Cobalt salt solution was introduced immediately after DCP. Different compositions of PP-UP blends are shown in Table 1.

\subsection{Apparatus and methodology}

Melt Flow Basic supplied by KARG Industrietechnik was used to measure the melt flow index of the blends. The reservoir diameter is $9.55 \mathrm{~mm}$ with different dies having equal diameter $(2.095 \mathrm{~mm})$ but different lengths of 4,10 and $30 \mathrm{~mm}$. The piston was inserted inside the reservoir carrying various loads and it moves inside the reservoir with precise tolerance between piston and reservoir wall. The experiment was conducted at a temperature of $200^{\circ} \mathrm{C}$ for different PP-UP compositions under different loads ranging from $1.6 \mathrm{~kg}$ to 7.0 $\mathrm{kg}$.

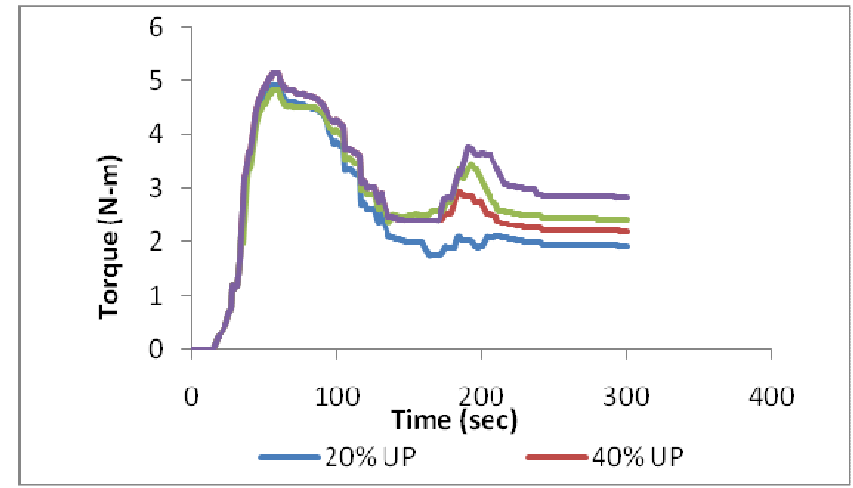

Figure 1: Torque as a function of time for different PP-UP blends

\section{Results and Discussion}

\subsection{Plastograph study}

The plastograph was studied to have an insight into processing characteristics. The changing trend of torque for different blend compositions over experimental range of time is shown in Figure 1.

Initially, the torque value is high because of un-melted granules of polypropylene. The torque value tends to decrease as the melting proceeds. At this stage, addition of unsaturated polyester reduces the torque sharply by providing lubrication to the melt. As the mixing proceeds, the value of torque tends to be stable. After 3 minutes, a sudden rise in torque is observed which corresponds to the grafting of unsaturated polyester due to addition of DCP followed by the cobalt salt solution. The degradation of PP also starts simultaneously but at a slower rate than grafting. It is clear from slight but steady decrease in torque value. When both the reactions are completed, the torque remains stable. It is evident from the graph that torque value is higher for blends with greater UP content which is an indication of increase in grafting.

\subsection{Rheological study}

Figure 2 represents Melt flow index (MFI) as a function of unsaturated Polyester concentration. It has been observed that MFI decreases with increase in UP concentration. MFI is dependent upon two competing reactions: first one is the peroxide initiated degradation of $\mathrm{PP}$, which tends to increase the MFI value. The second reaction is the coupling reaction of PP-UP which tends to reduce the MFI. It is evident from the fig. that MFI shows an overall decreasing trend with increase in UP concentration for all conditions of load. This decrease in MFI is a clear indication of dominance of grafting reaction over degradation reaction. It is also observed from the fig. that increase of unsaturated polyester concentration over $60 \%$ slightly effect the MFI value.

Figure 3 shows a linear relationship between apparent shear stress and shear rate on bi-logarithmic coordinate system. It implies that the melt shear flow obeys the power law relationship

$$
\tau=K \gamma_{a}^{n}
$$




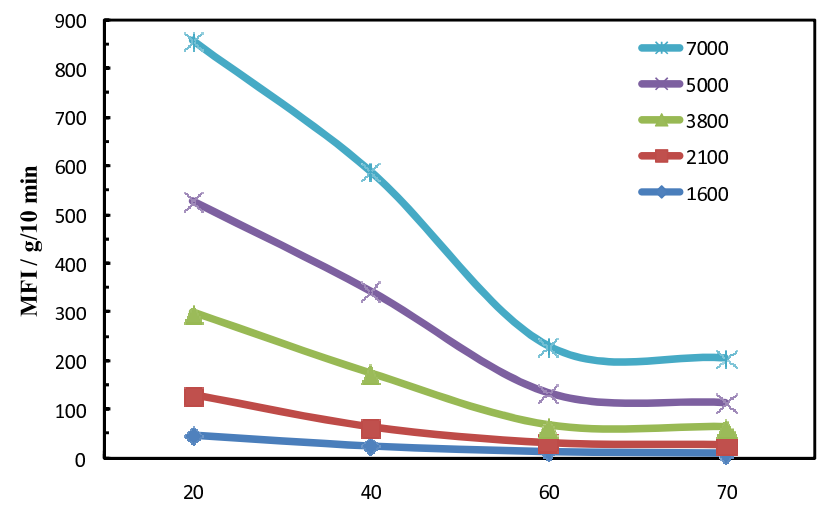

Figure 2: MFI as a function of UP concentration

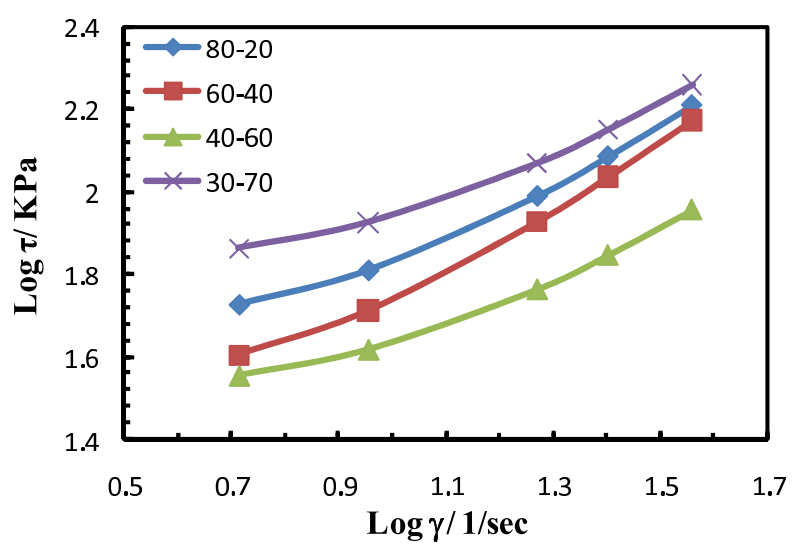

Figure 3: $\log \tau$ as a function of $\log \gamma$ at different UP concentration

where $K$ is the melt viscosity index and $n$ is the power law index.

From the calculated value of power law coefficients, it is clear that the value of $\mathrm{n}$ is lower for 30-70 composition and it is higher for 80-20 batch (Figure 4). It has been found that degraded PP exhibits newtonian behavior while the grafted PP-UP blend is inclined towards non-newtonian behavior [13]. The higher value of the 80-20 composition is attributed towards the more newtonian behavior of degraded PP. On the other hand, as UP concentration is increased up to a limit, the trend shifts from Newtonian to the non-newtonian behavior due to dominance of grafting reaction. The power law indices of these compositions also support this argument.

From Figure 5 it is evident that increase in UP content is not much effective in increasing consistency index at lower concentration. However, at higher UP content, there is a sharp increase in consistency index because of higher degree of grafting. At lower concentration of unsaturated polyester, the degradation of PP is governing reaction as indicated by the melt shear curves. Initially there is a slight increase in consistency index, i.e. lower force is required to flow it. But at higher UP concentration where grafting reaction becomes dominant, further increase in unsaturated polyester concentration will result in higher value of consistency index. It

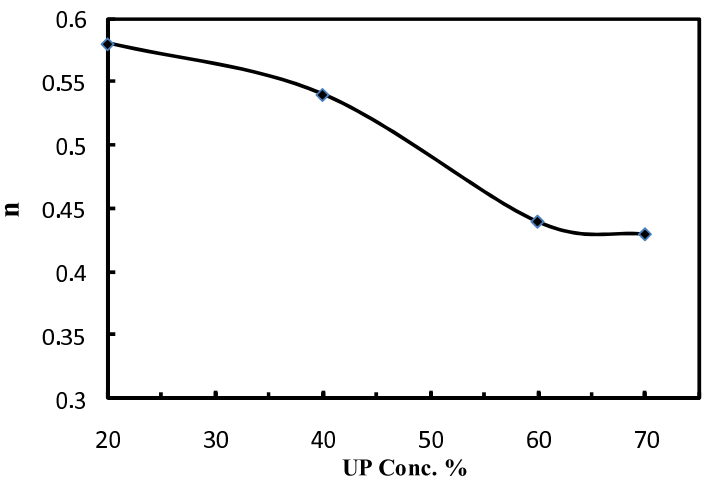

Figure 4: Power law index as a function of UP concentration

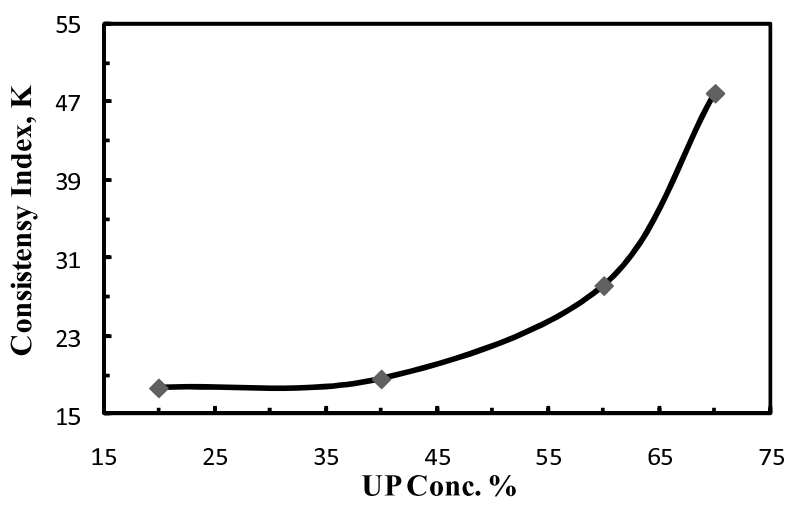

Figure 5: Consistency index as function of the UP concentration

implies that force required to flow the resultant material increases in magnitude [14].

Figure 6 shows the trend of apparent viscosity as a function of apparent shear rate for different compositions of the blend. It is implicit in the fig that viscosity reduces with increase in shear rate but the decrease in viscosity is quantitatively different for different compositions. This reduction in viscosity is because of alignment of chains in direction of force. From the curve, it is evident that 80-20 batch shows poor shear thinning behavior. This behavior can be attributed towards dominant PP degradation reaction. As unsaturated polyester concentration is increased, there will be more grafting reaction and it is supported by marked shear thinning behavior.

For the case of flow of polymer through circular die, a correction is normally applied to take into account pseudoplatic nature of the melt which implies that the assumed parabolic profile in the die actually illustrates more plug behavior [15]. This correction is generally expressed as

$$
\tau_{\text {true }}=\frac{4 Q}{\pi R^{3}} \frac{(3 n+1)}{4 n}
$$

True viscosity-strain rate curve obtained after applying correction for viscosity and strain rate is illustrated in the Figure 7. The curve is almost similar to the Figure 6 but the slop of curve for the case of $70 \%$ UP concentration changes 


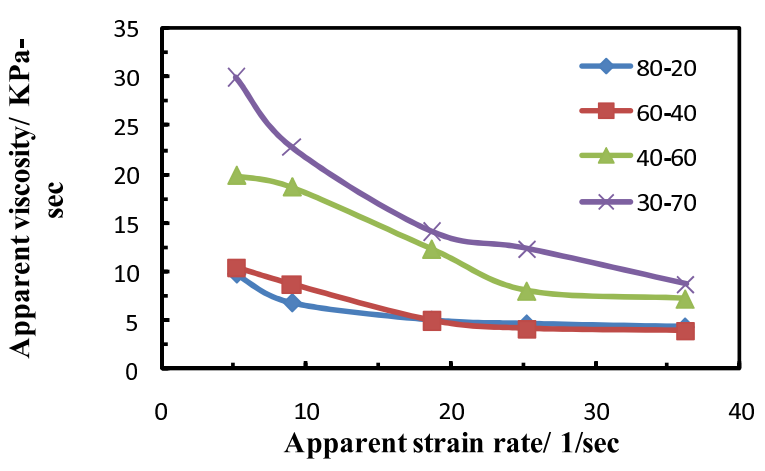

Figure 6: Apparent viscosity as a function of apparent strain rate at different composition

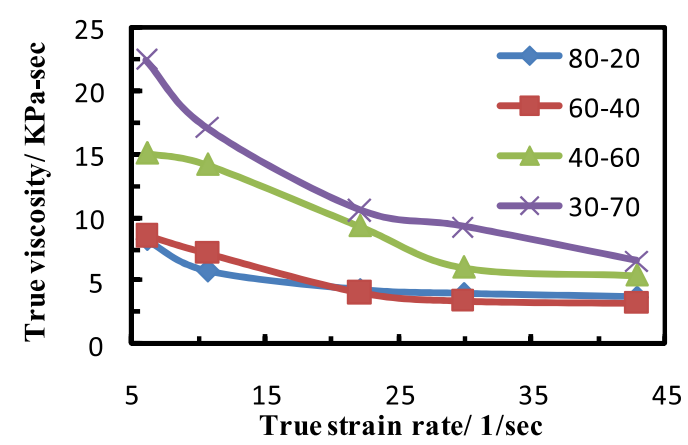

Figure 7: True strain rate as a function of the true viscosity at different UP concentration

slightly. This behavior is also followed by the $60 \%$ UP composition because of their proximity towards non-Newtonian behavior.

\section{Conclusion}

The increase in UP content in the blend results in a decrease in MFI value and its magnitude is dependent on the extent of grafting of UP on PP and degradation reaction of PP. Decrease in the value of power law index and increase in consistency index is also observed with increase in UP concentration. This behavior is associated with the pronounced shear thinning phenomenon.

\section{References}

[1] Fried JR, Polymer Science and Technology, Prentice-Hall, New Delhi, 1999

[2] Wan C, Patel SH and Xanthos M, Reactive melt modification of polypropylene with a crosslinkable polyester, Polymer Engineering and Science, 2003. 43(6):pp. 1276-1288. doi:10.1002/pen.10108

[3] Mackenzie MW, Polymeric film: US Patent 4771108, 1988

[4] Boon W and Henderson J, High performance rubber-polyester blends: US patent 4558096, 1985, uS Patent 4,558,096

[5] Joffrion R, Polar resins having improved characteristics by blending with EPM and EPDM polymers: US Patent 4251644, 1981

[6] Sunseri R and O'Rourke M, Polyesters having improved impact strength: US Patent 4977217, 1990
[7] Fink J, Reactive polymers fundamentals and applications: a concise guide to industrial polymers, PDL handbook series, William Andrew, 2005

[8] Carreau PJ, Rheological Equations from Molecular Network Theories, Journal of Rheology, 1972. 16(1):pp. 99-127. doi:10.1122/1.549276

[9] Cross MM, Rheology of non-Newtonian fluids: A new flow equation for pseudoplastic systems, Journal of Colloid Science, 1965. 20(5):pp. 417 - 437. doi:10.1016/0095-8522 (65) 90022-X

[10] Reiner M, Deformation, strain and flow: an elementary introduction to rheology, H. K. Lewis, 1960

[11] Vanderbilt J and Meyer Jr M, Polypropylene-graft-unsaturated polyester compositions and process for the production thereof: US Patent 5432230, 1995

[12] Liang JZ, Characteristics of melt shear viscosity during extrusion of polymers, Polymer Testing, 2002. 21(3):pp. 307 - 311. doi:10.1016/S0142-9418(01)00088-5

[13] Azizi H, Ghasemi I and Karrabi M, Controlled-peroxide degradation of polypropylene: Rheological properties and prediction of MWD from rheological data, Polymer Testing, 2008. 27(5):pp. 548 - 554. doi:10.1016/j.polymertesting.2008.02.004

[14] Dautant F, Simancas K, Sandoval A and Müller A, Effect of temperature, moisture and lipid content on the rheological properties of rice flour, Journal of Food Engineering, 2007. 78(4):pp. 1159 - 1166. doi:10.1016/j.jfoodeng.2005.12.028

[15] Cogswell F, Polymer melt rheology: a guide for industrial practice, George. Godwin in association with Plastics and Rubber Institute, 1981 